\title{
INFORMATION TECHNOLOGY INVESTMENT
}


This page intentionally left blank 


\section{INFORMATION}
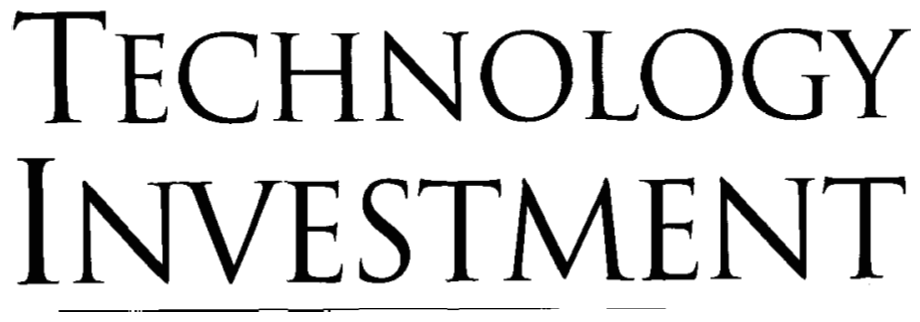

Decision-Making Methodology

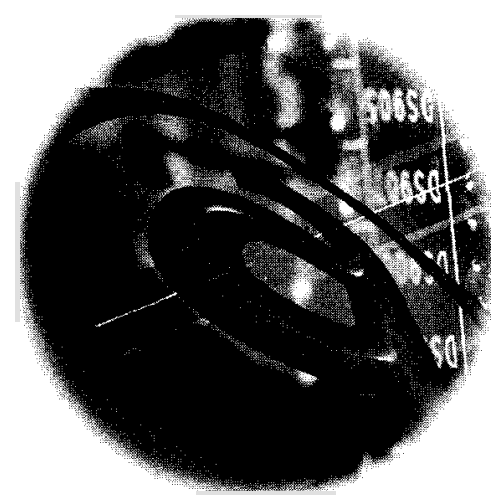

Marc J. Schniederjans University of Nebraska-Lincoln, USA

Jamie L. Hamaker University of Nebraska-Lincoln, USA Ashlyn M. Schniederjans Johns Hopkins University, USA 


\section{Published by}

World Scientific Publishing Co. Pte. Ltd.

5 Toh Tuck Link, Singapore 596224

USA office: 27 Warren Street, Suite 401-402, Hackensack, NJ 07601

UK office: 57 Shelton Street, Covent Garden, London WC2H 9HE

\section{British Library Cataloguing-in-Publication Data \\ A catalogue record for this book is available from the British Library.}

First published 2004

Reprinted 2005

\section{INFORMATION TECHNOLOGY INVESTMENT \\ Decision-Making Methodology}

Copyright $\odot 2004$ by World Scientific Publishing Co. Pte. Ltd.

All rights reserved. This book, or parts thereof, may not be reproduced in any form or by any means, electronic or mechanical, including photocopying, recording or any information storage and retrieval system now known or to be invented, without written permission from the Publisher.

For photocopying of material in this volume, please pay a copying fee through the Copyright Clearance Center, Inc., 222 Rosewood Drive, Danvers, MA 01923, USA. In this case permission to photocopy is not required from the publisher.

ISBN 981-238-695-5 
To my Myia, for your patience, understanding and support. 
This page intentionally left blank 


\section{Preface}

Periods of business activity are often marked by and referred to as "ages" in the historical development of the field of business. According to most business historians we have advanced from the "age of information" into the "age of knowledge". In both of these periods of time, information technology has been a determining factor in the survival and success of firms competing with one another. Those firms that know how to best invest in information technology have been and will continue to be the successors in this and future eras of business history.

Regardless of your position in an organization, investing in information technology may be the most important decision you will ever face in business. Unfortunately, investing in information technology is not as easy as common financial investment decisions. Careful consideration of financial and non-financial criteria may have to be included in the analysis to render an optimal solution. To make good decisions on information technology today requires the use of a variety of investment methodologies. These investment methodologies must be able to integrate the complexity of decision criteria in such a way that a decision choice is clear and clearly supported by the analysis. Today, just generating a decision is not enough. Information technology decisions must be supported by comprehensive inclusion of all relevant decision-making criteria.

The purpose of this textbook is to provide an in-depth treatment of a wide-variety of decision-making methodologies focused on the subject of investing in information technology. The methodological procedures as well as computer solutions to basic financial and advanced decision- 
making methodologies will be presented as tools for investing in information technology.

This textbook has been designed for an upper-level undergraduate course or a graduate business or engineering management course related to technology management for university students. Business faculty in areas of finance might also find this textbook useful for an applied investments course. Practitioners who work in information systems can also use this textbook if faced with a technology investment decisionmaking problem. Other groups of decision makers might include CEOs, vice presidents of information systems and finance, general managers, plant managers, supervisors, and industrial engineers. Other operations management and engineering faculty, trainers, and graduate students will also find this textbook present a useful variety of methodologies for managing and aiding information system investment decisions.

This textbook assumes that the reader has had some exposure to general mathematics and the terminology commonly found in business management. The basic technology/operations management or industrial management, and basic finance course that undergraduate students take for business degree programs, constitute sufficient prerequisite knowledge to satisfy the background to fully appreciate the content of this textbook. You do not have to be a technology manager or financial analyst to benefit from using this textbook. The terminology necessary to fully utilize this textbook is actually defined in the textbook. Also, throughout this textbook important terms are italicized and are usually followed by a definition. The location of the initial definitions can be found using the index at the end of the textbook.

The basic contents of this textbook are organized into twelve chapters consisting of four parts. In Part I, "Introduction to Information Technology Investment Decision-Making Methodology", three chapters are presented that help to define the basic subject and terminology used in the textbook, as well as briefly identifying the major topics that make up the rest of the textbook. In Part II, "Financial Information Technology Investment Methods", three chapters are devoted to explaining how basic financial methods are used and should be used in information technology decisions. In Part III, "Multi-Criteria Information Technology DecisionMaking Methods", the three chapters presented describe and illustrate a 
more complex set of decision-making methodologies that can be used individually or in combination with other methods to render information technology investment decisions. Finally, in Part IV, "Other Information Technology Investment Methods", a series of three additional chapters present a variety of other commonly used investment methodologies reported in the information technology literature. Collectively these chapters provide a comprehensive treatment of commonly used and more recently applied methodologies for technology investment decisionmaking. This textbook ends with an epilogue chapter focused on the issue of not making the right decision and how the consequences might be avoided.

M. J. Schniederjans J. Hamaker A. M. Schniederjans 
This page intentionally left blank 


\section{Contents}

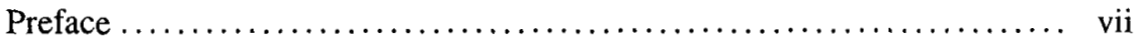

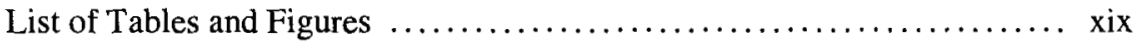

Part I Introduction to Information Technology Investment

Decision-Making Methodology

Chapter 1 Introduction to Information Technology Investment

Decision-Making 3

Introduction $\ldots \ldots \ldots \ldots \ldots \ldots \ldots \ldots \ldots \ldots \ldots \ldots \ldots \ldots \ldots \ldots \ldots \ldots, \quad 3$

Types of IT Investment Decision-Making Problems ....... 5

What are IT Investment and Decision-Making

Methodologies? ............................. 8

Limitations of Methodologies $\ldots \ldots \ldots \ldots \ldots \ldots \ldots \ldots \ldots, 10$

Why Study IT Investment and Decision-Making

Methodologies? ............................. 13

Organizational Strategic Planning in IT Investment

Decision-Making $\ldots \ldots \ldots \ldots \ldots \ldots \ldots \ldots \ldots \ldots \ldots \ldots, 14$

How This Book is Organized to Help You Learn .......... 21

Summary $\ldots \ldots \ldots \ldots \ldots \ldots \ldots \ldots \ldots \ldots \ldots \ldots \ldots \ldots, 24$

Chapter 2 Needs Analysis and Alternative Information Technology Investment Strategies

Introduction $\ldots \ldots \ldots \ldots \ldots \ldots \ldots \ldots \ldots \ldots \ldots \ldots \ldots \ldots \ldots \ldots \ldots, 29$

What is Needs Analysis? ......................... 31

Determining IT Processing Capacities and Specifications .... 35 
Alternative IT Investment Strategies $\ldots \ldots \ldots \ldots \ldots \ldots \ldots \ldots \quad 40$

What is IT Outsourcing? ............................ 42

An IT outsourcing strategy $\ldots \ldots \ldots \ldots \ldots \ldots \ldots, 45$

Final comment on IT outsourcing $\ldots \ldots \ldots \ldots \ldots, 46$

Summary $\ldots \ldots \ldots \ldots \ldots \ldots \ldots \ldots \ldots \ldots \ldots \ldots \ldots \ldots, 47$

Chapter 3 Measuring Information Technology Investment

Performance $\quad 53$

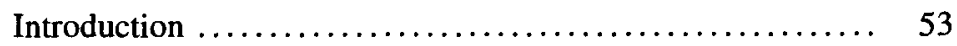

The Economics of Information ..................... 54

Examples of economic theories of information ..... 55

Why Measure IT Performance? ................... 57

Measures of IT Business Value $\ldots \ldots \ldots \ldots \ldots \ldots \ldots \ldots . \quad 59$

Financial Performance Measures of IT ............... 60

Business performance measures of IT .......... 62

Strategic performance measures of IT $\ldots \ldots \ldots \ldots, 64$

Costs of IT ............................. 65

Measures of IT effectiveness value ............ 66

Measures of IT efficiency value .............. 72

Summary $\ldots \ldots \ldots \ldots \ldots \ldots \ldots \ldots \ldots \ldots \ldots \ldots \ldots \ldots \ldots \ldots \ldots, 74$

Part II Financial Information Technology Investment Methods

$\begin{array}{lll}\text { Chapter } 4 \text { Basic Financial Methods } & 81\end{array}$

Introduction $\ldots \ldots \ldots \ldots \ldots \ldots \ldots \ldots \ldots \ldots \ldots \ldots \ldots \ldots, 81$

What are the Basic Financial Methods? ............... 81

What is Breakeven Analysis? .................... 83

A statistical approach to non-quantifiable benefits . 86

What is Payback Period Methodology?............... 88

What is Accounting Rate of Return Methodology? ........ 93

Summary $\ldots \ldots \ldots \ldots \ldots \ldots \ldots \ldots \ldots \ldots \ldots \ldots \ldots \ldots \ldots, \quad 97$

$\begin{array}{lll}\text { Chapter } 5 \text { Other Financial Methodologies } & 105\end{array}$

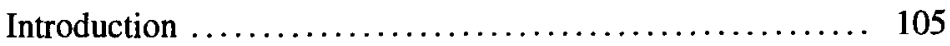


What Types of Methodologies Support IT Investment

Decision-Making? .......................... 106

Financial techniques for IT investment decision-making 107

Operations research/management science techniques for IT investment decision-making 108

Techniques specifically designed for IT investment decision-making

Other techniques for IT investment decision-making $\ldots \ldots \ldots \ldots \ldots \ldots \ldots \ldots \ldots \ldots$

What is Present Value Analysis Methodology? ........... 117

Net present value analysis methodology .......... 119

Computer-based solutions .................. 121

Unequal investment sizes $\ldots \ldots \ldots \ldots \ldots \ldots \ldots 122$

Unequal investment lives $\ldots \ldots \ldots \ldots \ldots \ldots \ldots \ldots 123$

What is Return on Investment Methodology? ............ 125

ROI strategy for enterprise resource planning systems

What is Internal Rate of Return? .................. 128

Computer-based solutions $\ldots \ldots \ldots \ldots \ldots \ldots \ldots, 131$

A Word About Cash Flows $\ldots \ldots \ldots \ldots \ldots \ldots \ldots \ldots \ldots, 132$

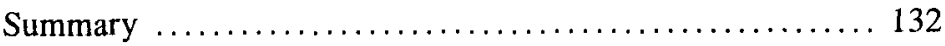

Chapter 6 Cost/Benefit Analysis 139

Introduction .................................. 139

What is Cost/Benefit Analysis? ...................... 140

Define problem ......................... 140

Identification and quantification of cost and benefit $\ldots \ldots \ldots \ldots \ldots \ldots \ldots \ldots \ldots \ldots \ldots \ldots, 142$

Compare alternatives $\ldots \ldots \ldots \ldots \ldots \ldots \ldots \ldots \ldots, 146$

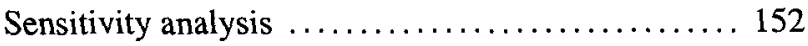

What is Cost/Effectiveness Analysis? ................. 154

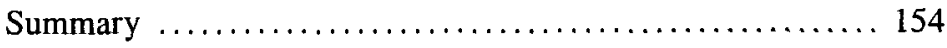


Part III Multi-Criteria Information Technology Decision-Making Methods

Chapter 7 Critical Success Factors, Delphi Method and the Balanced Scorecard Method

Introduction ................................... 163

What are Critical Success Factors? .................... 164

What is the Delphi Method? ...................... 170

What is the Balanced Scorecard Method? ............. 172

Balanced scorecard components ............... 173

Illustration of the balanced scorecard method ...... 180

Final word on balanced scorecards ............. 183

Summary $\ldots \ldots \ldots \ldots \ldots \ldots \ldots \ldots \ldots \ldots \ldots \ldots \ldots . . . \ldots \ldots$

Chapter 8 Multi-Factor Scoring Methods and the Analytic Hierarchy Process $\quad 189$

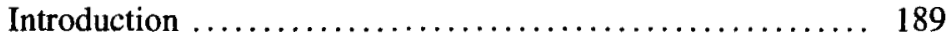

What are Multi-Factor Scoring Methods? ............. 190

Types of multi-factor scoring methods $\ldots \ldots \ldots \ldots 191$

Summary of multi-factor scoring methods solution procedures ......................... 194

Sensitivity analysis of multi-factor scoring methods .......................... 195

Spreadsheet and computer solutions ........... 197

What is the Analytic Hierarchy Process? ............. 198

The AHP procedure ....................... 199

Summary of the AHP solution procedure $\ldots . \ldots \ldots .221$

Spreadsheet and computer solutions ............ 221

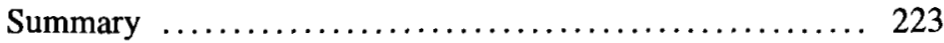

Chapter 9 Decision Analysis and Multi-Objective Programming Methods 233

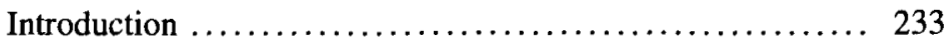

What is Decision Theory? ........................ 234

Decision theory problem/model elements ........ 234 
Types of decision environments .............. 236

Decision Theory Formulation and Solution Methodologies .. 237

A decision theory problem/model formulation procedure

Decision-Making Under Certainty $\ldots \ldots \ldots \ldots \ldots \ldots \ldots .240$

Maximax criterion ....................... 241

Maximin criterion ........................... 241

Decision-Making Under Risk ..................... 242

Origin of probabilities $\ldots \ldots \ldots \ldots \ldots \ldots \ldots \ldots, 243$

Expected value criterion $\ldots \ldots \ldots \ldots \ldots \ldots \ldots \ldots, 244$

Expected opportunity loss criterion ............. 244

Decision-Making Under Uncertainty ................ 245

Laplace criterion .......................... 247

Maximin criterion $\ldots \ldots \ldots \ldots \ldots \ldots \ldots \ldots \ldots, 248$

Maximax criterion .......................... 249

Hurwicz criterion ......................... 249

Minimax criterion .......................... 249

Multi-Objective Programming Methodology ........... 251

What is Goal Programming? ...................... 253

Goal programming problem/model elements ...... 253

Goal Programming Problem/Model Formulation .......... 263

GP problem/model formulation procedure ....... 263

GP problem/model formulation problems ........ 265

Computer-based solutions for goal programming .. 272

Goal Programming Complications and Model Assumptions .. 276

GP complications ....................... 276

GP model assumptions ...................... 278

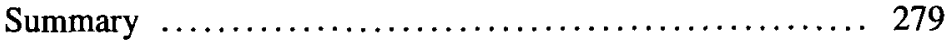

Part IV Other Information Technology Investment Methods

Chapter 10 Benchmarking Techniques and Game Theory 289

Introduction .................................... 289

What is Benchmarking? ........................ 290 
A benchmarking procedure $\ldots \ldots \ldots \ldots \ldots \ldots \ldots 292$

A benchmarking methodology: Gap analysis ..... 294 What is Game Theory? ........................... 296

Basic structure of game theory problem/model ... 297

Objectives and assumptions ................. 299

Game theory problem/model formulation procedure ............................ 300

Solution Procedures for Game Theory Problems ......... 304

Rational choice method: A saddle point solution ... 304

The minimax solution method ................. 309

A Minimax Solution for a Mixed Strategy Problem ........ 311

A computer-based solution for a mixed strategy

problem ........................... 313

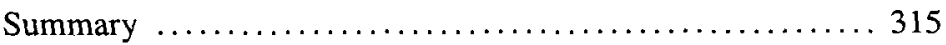

Chapter 11 Investment Portfolio Methodologies 321

Introduction .................................... 321

What are Investment Portfolio Methodologies? ......... 322

Ward's Portfolio Approach . ...................... 322

Evaluating individual investments $\ldots \ldots \ldots \ldots \ldots, 327$

Setting priorities ......................... 328

Investment Mapping Methodology $\ldots \ldots \ldots \ldots \ldots \ldots \ldots \ldots, 334$

Mapping the investment portfolio ............. 335

Evaluating IT investments $\ldots \ldots \ldots \ldots \ldots \ldots \ldots, 339$

Managing benefits ........................ 343

Other Investment Portfolio Methodologies .............. 344

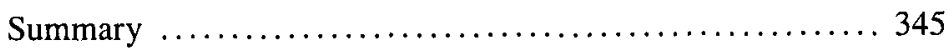

Chapter 12 Value Analysis and Benefit/Risk Methodologies 349

Introduction ...................................... 349

What is Value Analysis Methodology? ............... 350

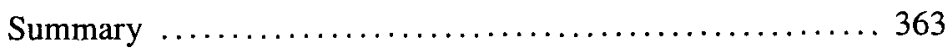


Epilogue: The Costs of Not Making the Right Information

Technology Decision (and Strategies on

How to Avoid Them) $\quad 367$

Macro Economic View ............................ 367

Micro Economic View .......................... 369

Strategies for Making the Right IT Investment

Decisions and Avoiding IT Costs ................ 374

"So What's the Good News?" ....................... 377

$\begin{array}{ll}\text { Index } & 383\end{array}$ 
This page intentionally left blank 


\section{Lists of Tables and Figures}

\section{TABLES}

\section{Chapter 1}

Table 1. PC selection criteria and measures $\ldots \ldots \ldots \ldots \ldots \ldots \ldots, 6$

Table 2. Select IT methodologies $\ldots \ldots \ldots \ldots \ldots \ldots \ldots \ldots \ldots \ldots \ldots \ldots \ldots \ldots$

Table 3. System implementation strategies $\ldots \ldots \ldots \ldots \ldots \ldots \ldots \ldots .20$

\section{Chapter 2}

Table 1. Costs considerations in the needs analysis report $\ldots \ldots \ldots \ldots, 35$

Table 2. Alternative IT investment strategies $\ldots \ldots \ldots \ldots \ldots \ldots \ldots, 41$

Table 3. Outsourcing alternative strategies $\ldots \ldots \ldots \ldots \ldots \ldots \ldots \ldots, 43$

\section{Chapter 3}

Table 1. Financial performance measures ..................6 61

Table 2. Business performance measures from the customer perspective $\ldots \ldots \ldots \ldots \ldots \ldots \ldots \ldots \ldots \ldots \ldots \ldots \ldots \ldots \ldots . \ldots \ldots$

Table 3. Business performance measures from the internal perspective .. 63 Table 4. Business performance measures from the innovation and learning perspective $\ldots \ldots \ldots \ldots \ldots \ldots \ldots \ldots \ldots \ldots, 64$

Table 5. Business performance measures from the financial perspective . 64 Table 6. Cost categorizations for IT $\ldots \ldots \ldots \ldots \ldots \ldots \ldots \ldots \ldots \ldots \ldots \ldots$

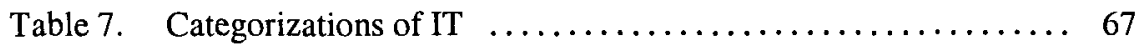

Table 8. Effectiveness measures for IT to support business processes ... 68

Table 9. IT effectiveness factors $\ldots \ldots \ldots \ldots \ldots \ldots \ldots \ldots \ldots \ldots . \ldots \ldots$

Table 10. Effectiveness measures for IT to support employees ........ 70

Table 11. Effectiveness measures for IT sourcing $\ldots \ldots \ldots \ldots \ldots \ldots \ldots 71$

Table 12. Additional effectiveness measures for IT sourcing $\ldots \ldots \ldots \ldots 73$ Table 13. Efficiency measures for IT sourcing $\ldots \ldots \ldots \ldots \ldots \ldots \ldots \ldots$ 


\section{Chapter 4}

Table 1. Use of financial methodologies in IT evaluation $\ldots \ldots \ldots \ldots .83$

Table 2. Costs and benefits of a computer system example ......... 85

Table 3. Present value calculations for the computer system example ... 85

Table 4. Sample data for absenteeism example $\ldots \ldots \ldots \ldots \ldots \ldots \ldots 88$

Table 5. Data for a payback period problem ................. 89

Table 6. Data for a payback period revised problem .............. 90

Table 7. Results for payback period problem ................. 90

Table 8. Data for alternative payback period example ............ 91

Table 9. Results for a revised payback period problem $\ldots \ldots \ldots \ldots \ldots, 92$

Table 10. Average annual net income data .................. 94

Table 11. Average annual book value of assets data $\ldots \ldots \ldots \ldots \ldots \ldots 95$

Table 12. Revised average annual net income data .............. 96

Table 13. Revised average annual book value of assets data ......... 96

\section{Chapter 5}

Table 1. Financial techniques for IT investment decision-making . . . . 109

Table 2. Operations research/management science techniques for IT investment decision-making $\ldots \ldots \ldots \ldots \ldots \ldots \ldots \ldots \ldots$

Table 3. Techniques specifically designed for IT investment decision-making ........................... 112

Table 4. Other techniques for IT investment decision-making $\ldots \ldots \ldots 115$

Table 5. Data for a present value analysis problem for mutually exclusive investments ....................... 120

Table 6. Data for a present value analysis problem with mutually exclusive investments ....................... 120

Table 7. Data for a present value analysis problem with unequal

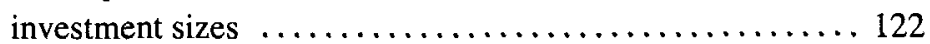

Table 8. Profitability index for a present value analysis problem with

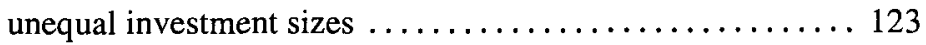

Table 9. Data for a present value analysis problem with unequal investment lives ........................... 124

Table 10. NPV replicated to a common denominator $\ldots \ldots \ldots \ldots \ldots \ldots 124$

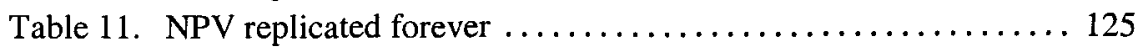

Table 12. Data for an internal rate of return problem .............. 129

Table 13. Data for conflicting internal rate of return and net present

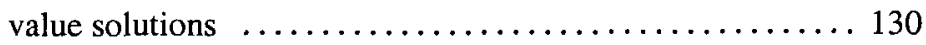




\section{Chapter 6}

Table 1. Potential costs of an IT investment ................. 143

Table 2. Potential benefits of an IT investment ................. 145

Table 3. Costs and benefits of Computer System A .............. 148

Table 4. Costs and benefits of Computer System B .............. 148

Table 5. Present value of costs and benefits for Computer System A . . . 148

Table 6. Present value of costs and benefits for Computer System B ... 149

Table 7. Common criteria to evaluate IT investments in cost/benefit

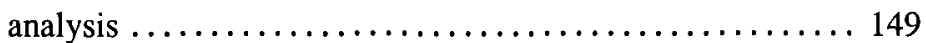

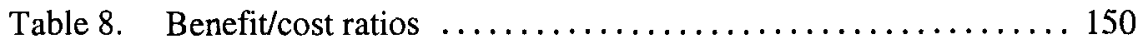

Table 9. Net present value of net benefits for Computer Systems

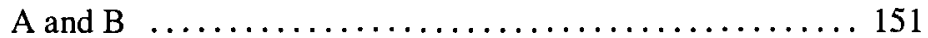

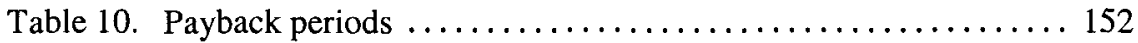

\section{Chapter 7}

Table 1. Criteria useful in determining CSFs ................. 167

Table 2. Examples of CSFs relationship with strategic goals $\ldots \ldots \ldots \ldots 168$

Table 3. Steps in the Delphi method for CSF consensus building $\ldots \ldots 171$

Table 4. Example of financial perspective scorecard for developing

IT projects ............................... 175

Table 5. Example of customer perspective scorecard for developing

IT projects ........................... 177

Table 6. Example of the internal business perspective scorecard for developing IT projects ...................... 179

Table 7. Example of the learning and growth perspective scorecard for developing IT projects $\ldots \ldots \ldots \ldots \ldots \ldots \ldots \ldots \ldots \ldots \ldots$

\section{Chapter 8}

Table 1. Multi-factor scoring method table for un-weighted problem $\ldots .191$ Table 2. Multi-factor scoring method table for weighted problem ...... 193

Table 3. Computation for the multi-factor scoring method weighted problem ................................. 193

Table 4. Computations for revised parameter MFSM sensitivity analysis problem .......................... 197

Table 5. Excel ${ }^{\circledR}$ printout of the Excel® formulas used in the MFSM problem

Table 6. Excel@ printout of un-weighted and weighted 
Table 7. Initial AHP Step 2 comparison table for computer selection

problem ................................ 202

Table 8. Diagonal comparisons AHP Step 2 pairwise table for

computer selection problem .................. 203

Table 9. Between comparisons AHP Step 2 pairwise table for

computer selection problem ................... 204

Table 10. Complete comparison AHP Step 2 pairwise table for

computer selection problem ................... 205

Table 11. Diagonal comparison AHP table for brand name factor ...... 205

Table 12. Between comparison AHP table for brand name factor ...... 206

Table 13. Complete comparison AHP table for brand name factor $\ldots \ldots 206$

Table 14. Diagonal comparison AHP table for price factor .......... 207

Table 15. Between comparison AHP table for price factor .......... 207

Table 16. Complete comparison AHP table for price factor $\ldots \ldots \ldots \ldots 207$

Table 17. Complete comparison AHP table for delivery factor ........ 208

Table 18. Step 3 decimals and summation of all AHP tabled values ..... 209

Table 19. Step 3 ratios of column total AHP tabled values $\ldots \ldots \ldots \ldots 210$

Table 20. Step 3 Final substep AHP priority calculations $\ldots \ldots \ldots \ldots 212$

Table 21. Initial AHP comparison table for factor weights $\ldots \ldots \ldots \ldots 213$

Table 22. Diagonal comparisons AHP pairwise table for factor weights .. 213

Table 23. Between comparisons AHP pairwise table for factor weights ... 214

Table 24. Complete comparison AHP pairwise table for factor weights ... 214

Table 25. Decimal and summation of AHP column tabled values $\ldots \ldots \ldots 215$

Table 26. Ratios of column total AHP tabled values .............. 215

Table 27. Final AHP factor weight priority calculations $\ldots \ldots \ldots \ldots \ldots 216$

Table 28. Overall decision priority calculations $\ldots \ldots \ldots \ldots \ldots \ldots \ldots 217$

Table 29. Matrix multiplication computations for weighted sum vector $\ldots 218$

Table 30. Matrix multiplication computations for weighted sum vector . . 219

Table 31. Random index (RI) values for given " $n$ " number of

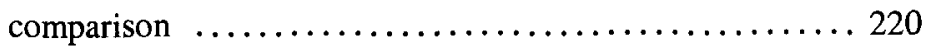

Table 32. Excel@ printout of AHP Step 3 Calculations $\ldots \ldots \ldots \ldots \ldots 222$

Table 33. Excel® printout of formulas used in AHP Step 3 calculations .. 223

\section{Chapter 9}

Table 1. Generalized statement of the DT problem/model .......... 235

Table 2. DT formulation of the network topology problem $\ldots \ldots \ldots \ldots 239$

Table 3. Formulation of new IT service product problem $\ldots \ldots \ldots \ldots 240$

Table 4. Maximax solution for DT network topology problem ....... 241 
Table 5. Maximin solution for DT network topology problem $\ldots \ldots \ldots 242$

Table 6. Expected value solution for DT network topology problem .... 245

Table 7a. Step 1 of expected opportunity loss solution for DT network

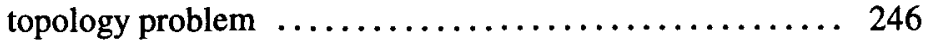

Table 7b. Steps 2 and 3 of expected opportunity loss solution for DT network topology problem $\ldots \ldots \ldots \ldots \ldots \ldots \ldots \ldots 247$

Table 8. Laplace solution for DT network topology problem ........ 248

Table 9. Hurwicz solution to the DT network topology problem ...... 250

Table 10. Minimax solution of the DT network topology problem ..... 252

Table 11. A generalized statement of a GP model .............. 254

Table 12. Selecting deviation variables for the GP objective function ... 256

Table 13. The different types of GP model objective functions . . . . . 260

Table 14. Data for LAN leasing problem $\ldots \ldots \ldots \ldots \ldots \ldots \ldots \ldots 265$

Table 15. GP model formulation of the LAN leasing problem $\ldots \ldots \ldots 268$

Table 16. GP model formulation of the outsourcing service problem $\ldots 272$

\section{Chapter 10}

Table 1. Benchmarking measures in IT ................... 291

Table 2. Typical customer questions for a gap analysis ........... 295

Table 3. General statement of the two-person, zero-sum game theory problem $/$ model .......................... 298

Table 4. Applied example of a GT problem/model formulation ....... 299

Table 5. GT problem/model formulation of IT ROI investment

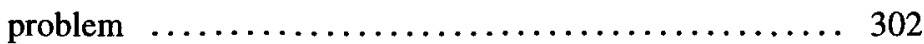

Table 6. GT problem/model formulation of IT ROI investment

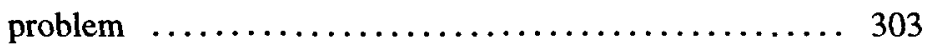

Table 7. Revised GT problem/model formulation of IT ROI investment

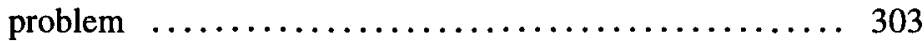

Table 8. First step in GT IT ROI investment problem solution ....... 305

Table 9. Second step in GT IT ROI investment problem solution ...... 306

Table 10. Third step in GT IT ROI investment problem solution ...... 306

Table 11. Fourth step in GT IT ROI investment problem solution ...... 307

Table 12. Solution if Player B moves first in IT ROI investment

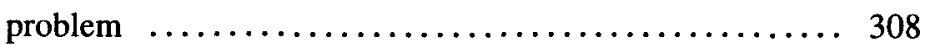

Table 13. Dominance in the GT IT ROI investment problem strategies $\quad . .309$

Table 14. Steps in using minimax solution method for the IT ROI

investment problem ........................ 310

Table 15. Formulation of the IT market share investment GT problem ... 312 
Table 16. Steps using minimax solution method for the IT market share investment problem

\section{Chapter 11}

Table 1. Priorities for a strategic IT investment problem ........... 329

Table 2. Multi-factoring scoring method for factory IT investments ... 331

Table 3. Priorities for a factory IT investment problem $\ldots \ldots \ldots \ldots \ldots 332$

Table 4. Priority rules for each IT investment category $\ldots \ldots \ldots \ldots . \ldots 334$

\section{Chapter 12}

Table 1. Sample of "size" risk assessment questions $\ldots \ldots \ldots \ldots \ldots 35$

Table 2. Sample of "structure" risk assessment questions . .......... 359

Table 3. Sample of "technology" risk assessment questions $\ldots \ldots \ldots \ldots 360$

Table 4. Delivery and enablement risk questions based on

Currie (2003) performance indicators ............... 361

Table 5. Management and operations risk questions based on

Currie (2003) performance indicators .............. 362

Table 6. Client/vendor relationship risk questions based on

Currie (2003) performance indicators ............... 363

\section{Epilogue}

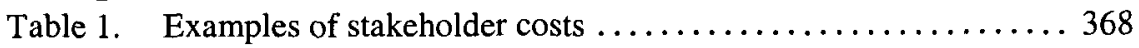

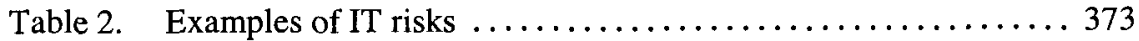

Table 3. Possible bias in our decision process $\ldots \ldots \ldots \ldots \ldots \ldots \ldots . \ldots \ldots$

\section{FIGURES}

\section{Chapter 1}

Figure 1. Sequential PC selection process $\ldots \ldots \ldots \ldots \ldots \ldots \ldots \ldots \ldots, 7$

Figure 2. Management information system (MIS) $\ldots \ldots \ldots \ldots \ldots \ldots \ldots$

Figure 3. MIS hierarchical planning stages $\ldots \ldots \ldots \ldots \ldots \ldots \ldots \ldots 16$

Figure 4. Detailed MIS hierarchical planning of IT systems $\ldots \ldots \ldots \ldots 18$

\section{Chapter 2}

Figure 1. Tactical MIS planning process for IT projects $\ldots \ldots \ldots \ldots \ldots 30$

Figure 2. Detailed steps of tactical MIS planning process for 
Figure 3. Reengineering procedure $\ldots \ldots \ldots \ldots \ldots \ldots \ldots \ldots \ldots \ldots, 37$

Figure 4. A process flowchart (before reengineering is applied) $\ldots \ldots \ldots 38$

Figure 5. A process flowchart (after reengineering is applied) $\ldots \ldots \ldots, 39$

\section{Chapter 3}

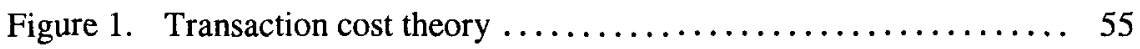

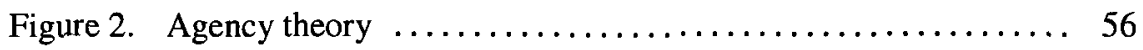

Figure 3. Example of process/IT matrix $\ldots \ldots \ldots \ldots \ldots \ldots \ldots \ldots, 68$

\section{Chapter 6}

Figure 1. The five stages of cost/benefit analysis $\ldots \ldots \ldots \ldots \ldots \ldots \ldots 141$

\section{Chapter 7}

Figure 1. Strategic steps in MIS hierarchical planning of IT systems $\ldots . .165$

Figure 2. Polling company managers to determine company-wide

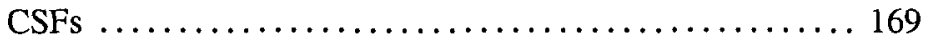

Figure 3. Overview of the balanced scorecard's four perspectives $\ldots . .174$ Figure 4. Leading and lagging indicator continuum in the balanced scorecard method and example $\ldots \ldots \ldots \ldots \ldots \ldots \ldots \ldots 18 \ldots \ldots \ldots$

\section{Chapter 8}

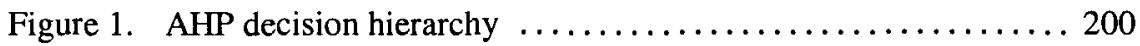

Figure 2. Decision hierarchy for computer system selection problem .... 201

\section{Chapter 9}

Figure 1. Computer solution for LAN leasing problem ........... 273

Figure 2. Computer solution for LAN leasing problem $\ldots \ldots \ldots \ldots \ldots 275$

\section{Chapter 10}

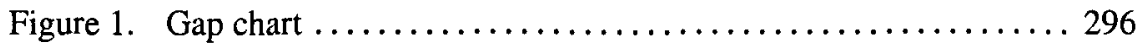

Figure 2. Computer solution for the IT market share investment

GT problem ............................ 314

\section{Chapter 11}

Figure 1. A Peters (1989) type benefits continuum ............... 336

Figure 2. A Peters (1989) type investment orientation continuum ...... 338

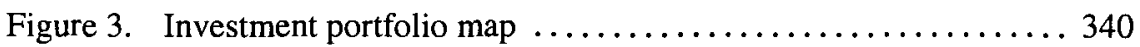

Figure 4. A Peters (1989) cost - benefit hierarchy .............. 342 
xxvi Information Technology Investment: Decision-Making Methodology

\section{Chapter 12}

Figure 1. A McFarlan and McKenney (1983) type project risk grid .... 357

\section{Epilogue}

Figure 1. Steps in MIS hierarchical planning of IT systems .......... 370

Figure 2. Detailed steps of tactical MIS planning process for

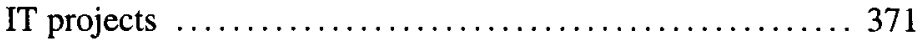

